\title{
Rendering stiffer walls: A hybrid haptic system using continuous and discrete time feedback
}

\author{
${ }^{1}$ Hari Vasudevan, $\quad{ }^{2}$ Manohar B.S $\quad$ and $\quad{ }^{1,3}$ M.Manivannan \\ ${ }^{1}$ Indian Institute of Technology Madras, Chennai, India, hvasudevan@smail.iitm.ac.in \\ ${ }^{2}$ Indian Institute of Science, Bangalore, India, bs.manohar@gmail.com \\ ${ }^{3}$ Massachusetts Institute of Technology, Cambridge MA, USA, mani@mit.edu
}

\begin{abstract}
Instability in conventional haptic rendering destroys the perception of rigid objects in virtual environments. Inherent limitations in the conventional haptic loop restrict the maximum stiffness that can be rendered. In this paper we present a method to render virtual walls that are much stiffer than those achieved by conventional techniques. By removing the conventional digital haptic loop and replacing it with a part-continuous and part-discrete time hybrid haptic loop, we were able to render stiffer walls. The control loop is implemented as a combinational logic circuit on an FPGA. We compared the performance of the conventional haptic loop and our hybrid haptic loop on the same haptic device and present mathematical analysis to show the limit of stability of our device. Our hybrid method removes the compute intensive haptic loop from the CPU, this can free a significant amount of resources that can be used for other purposes such as graphical rendering and physics modeling. It is our hope that in the future, similar designs will leads to a haptics processing unit or HPU.

keywords: Haptics, Instability ,Passiveness, Continuous Time
\end{abstract}

\section{INTRODUCTION}

Haptics has traditionally followed the developments and algorithms in the field of computer graphics to solve various issues that are common to both disciplines. However there are certain problems that are unique to haptics, one of them is the stability of contact interactions. Haptics by its very nature involves bidirectional interaction with the user: forces rendered by the haptic device and those exerted by the user are interdependent. Forces are displayed to the user by a "haptic loop". The haptic loop is a closed loop control system that determines the nature of interaction of the haptic device with the user based on the position and velocity of the end effector. Such a control system describes an impedance based haptic device. 
Most of the haptic devices use a digital haptic loop, which inherently suffers from quantization both temporally and spatially. This limits the maximum stiffness of the virtual surface that can be rendered. On contact with the virtual surface, the force rendered is very often controlled by the force law $F=k \Delta x-B v . \Delta x$ is the depth of penetration into the surface and ' $v$ ' is the velocity of the haptic device. The multiplier ' $k$ ' controls the stiffness of the virtual surface being rendered. In order to render a rigid surface, we would like as high a stiffness possible. However arbitrarily increasing the gain ' $k$ ' results in non-passivity of the haptic loop. This manifests itself as buzzing of the haptic device and destroys the realism of the environment.

We present new method of implementation of the haptic loop that increases the maximum stiffness that can be achieved by the haptic device. We eliminate the temporal quantization present in the conventional haptic loop by running it in continuous time. Another advantage of our hybrid system is that it frees the computer from the typical update rate $(1 \mathrm{Khz})$ of the rendered haptic force. This significantly frees the computational resources for other tasks such as graphics.

In the next section we present a brief review of the literature on the stability haptic loops. We then describe our system and compare its design with the conventional haptic loop. We then proceed to describe the implementation of the system and present the experimental results. This is followed by a short discussion on results and future work planned.

\section{Previous Work}

Literature on the stability of haptic devices has been mostly limited to the analysis and modification of digital haptic loops. [1] investigated the stability of Haptic interaction and derived a condition for the stability of the haptic device based on considerations of sampling rate of the controller. [2] extended this criterion to include the effects of position quantization. Their treatment of the instability problem involved the coulomb friction present in the haptic device.[3] recognize that passive analog control laws need not necessarily translate to stable digital control laws and derive conditions for such an analog

control law to successfully be transformed into a stable digital one. [4] describe energy leaks caused due to the Zero Order Hold inherent in digitally sampled control systems and present control strategies to create the illusion of a passive system to the user.[5] presented a theoretical analysis of the passivity of the stiff wall and in 2004, [6] provided a condition the haptic device must satisfy to exhibit passive behavior. [7] present a "Passivity Observer" and "Passivity Controller" method to track energy movements in haptic interactions with the user and to dissipate the excess energy if it tends to cause active behavior in the system. [8] explore the use of a multi-rate controller to reduce the $\mathrm{ZOH}$ effect in haptic devices and present a mathematical analysis of the same. A more recent paper by [9] accounts for inertia, viscous, and Coulomb friction of the device to the amplifier delay, sampling rate, encoder resolution, and controller stiffness. It also delineates areas of passive, locally stable, limit cycles and unstable behavior of the haptic device. The work on continuous time haptic devices are far and few in between, [10] describe a haptic interface device with an analog circuit which exerts continuous-time impedance 


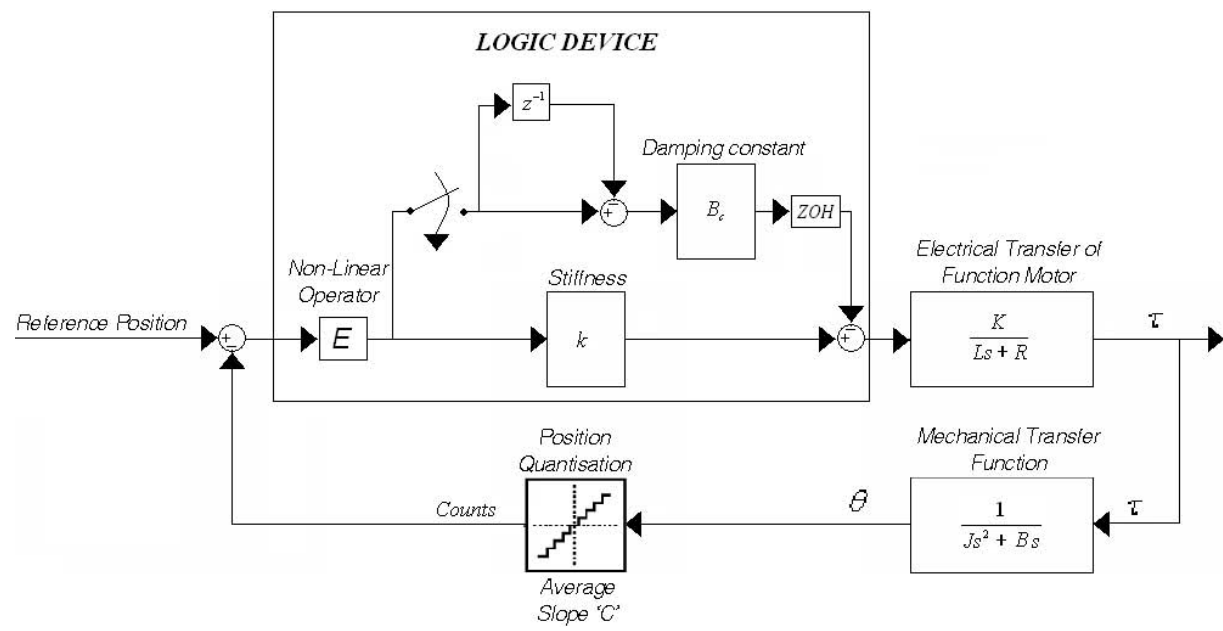

Figure 1: Diagram of our haptic control loop

within the sampling period of a conventional haptic loop. Though a continuous time circuit is used, it serves only to enhance the conventional digital loop. The continuous time loop is implemented using noise prone analog amplifiers and potentiometers. In contrast, our device makes use of asynchronous digital circuitry eliminating most noise problems. Our hybrid control loop also has the advantage that it eliminates the conventional haptic loop from the CPU. [11] seek to exploit the electrical characteristics of a DC motor to render virtual surfaces and interface it to virtual environments by means of "wave variables" and analog circuits alone. In our paper instead of exploiting the electrical characteristics of a DC motor, we use asynchronous digital circuits along with synchronous digital circuits which are readily available to obtain a comparable virtual wall stiffness.

\section{$3 \quad$ Stability of Haptic Devices}

Current haptic devices implement a discretised version of the force law: $F=k \Delta x-B v$. As [3] explain, discretizing this force law need not result in a stable haptic loop. [1] express the stability of the discrete force law as the upper limit on the maximum stiffness that can be achieved by a virtual wall. This expression takes the form : $k \leq \frac{2 B}{T}$ where ' $T$ ' is the sampling period of the control loop. [2] derive a condition of passivity of the virtual wall considering the amplitude quantization in the measurement of position and state the limit of stability as $k \leq \min \left\{\frac{2 B}{T}, \frac{2 f_{c}}{\Delta}\right\}$ where ' $f_{c}$ ' represents coulomb friction and ' $\Delta$ ' represents the minimum measurable change in position.

The above conditions for stability apply only to digital control loops. We will be able to achieve higher values of ' $k$ ' and hence stiffer walls if we employ an analog control system to control interactions between the user and virtual environments. True analog control systems are continuous in both time and amplitude. However the continuity in amplitude comes at a price - noise. Noise limits the performance of any analog control system. It is seen that all continuous time implementation attempted so far [10] and [11] involve analog circuitry. If we can eliminate the noise problems we might be able to develop a 
high performance analog control loop for haptic devices.

We have designed and fabricated a rotational haptic device and we attempt to implement the control law $\tau=k \Delta \theta$ in continuous time but with discrete amplitude feedback. Due to the rotational nature of the device, all linear parameters considered for the analysis of haptic devices have been replaced by their rotational counterparts. $\tau, \theta, \omega$ represent the torque, angular position and angular velocity respectively. The second part of the control law $B \omega$ is evaluated as done traditionally by sampling at various frequencies. We then proceed to implement the full control law $\tau=k \Delta \theta-B \omega$ as a combination of continuous and discrete time systems. It is seen that this system is capable of rendering much stiffer than it is possible with the traditional sampled data control system. Figure 1 shows the block diagram of the haptic control loop that has been implemented. E is a nonlinear operator given by:

$$
E= \begin{cases}1, & \text { Input } \leq 0 \\ 0, & \text { Input }>0\end{cases}
$$

Here constants $K, L, R$ are the Torque Constant, Inductance, Resistance of the motor respectively. The quantities $J, B, \tau$ are the Moment of Inertia, Mechanical Damping and Torque of the motor respectively.

\section{Experimental Haptic Device}

\subsection{Our Haptic device}

The haptic device consists of a Maxon RE29MAX - 226792 motor coupled to an 1024 cpr encoder. The shaft of the motor drives a larger wheel giving us a seven fold torque magnification. A picture of the experimental setup is shown in Figure 2.

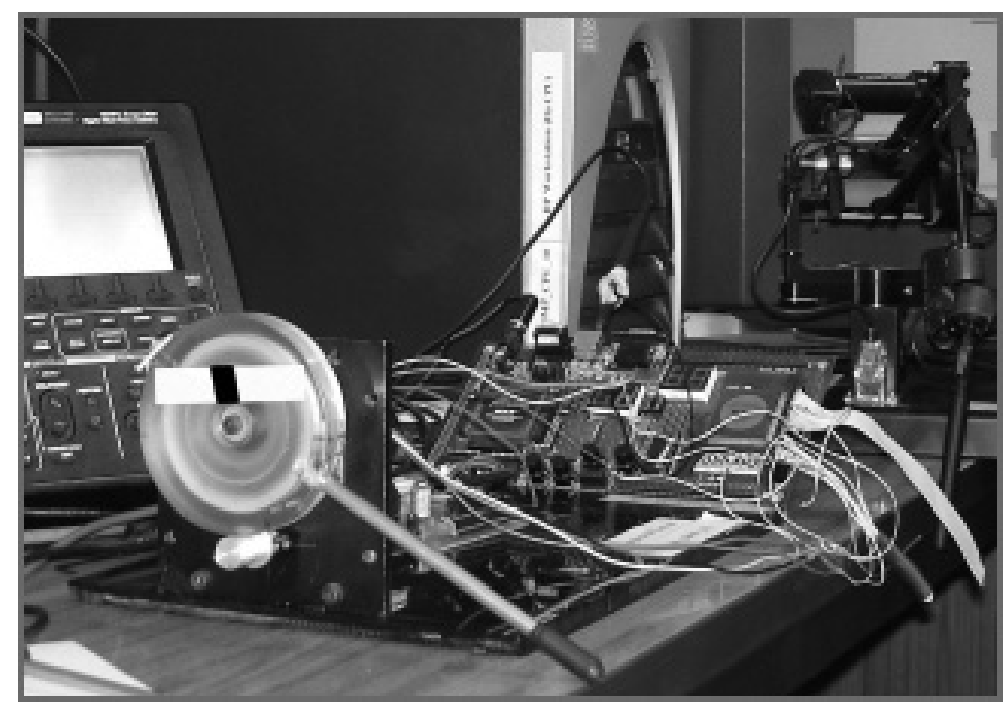

Figure 2: Picture of the haptic device 
The control loop is completed using an Altera-Flex10K20RC240-4 FPGA. The control equation is evaluated in two parts both of which are evaluated simultaneously. We evaluate

$$
\tau_{1}=k \Delta \theta
$$

where $\Delta \theta=\left(\theta-\theta_{\text {ref }}\right)$ Here $\theta$ is the position of the motor shaft obtained from the quadrature signals from the encoder. $\theta_{\text {ref }}$ is the position of the virtual wall which is specified by the user. The second part of the equation,

$$
\tau_{2}=B \omega
$$

is evaluated using simple backward differences. The Equations 2 and 3 are evaluated using single step(no clock) digital multipliers. All numbers are held in 12 bit locations. The result of any multiplication is therefore a 24 bit result. Since the motor control, pulse width modulator, has a maximum resolution of 12 bits, we truncate the result by eliminating the higher 12 bits. This does not affect the performance of the device because it was seen that the result often occupied only the lower 12 bits.

It is important to note that Equation 2 is evaluated asynchronously and it is this mode of operation that enables our device to render stiffer walls than is possible with conventional loops. In the course of our investigation we found that it was not possible to evaluate a velocity term asynchronously from position data. Hence Equation 3 requires sampled data. The velocity is therefore obtained by backward differences by evaluating

$$
\omega=\theta_{n}-\theta_{n-1}
$$

The characteristic division by the sampling period $T$ is not performed as Equation 4 is adequate to obtain a velocity estimate. In a final step we combine the results of Equation 2 and Equation 3 to form

$$
\begin{aligned}
\tau & =\tau_{2}+\tau_{3} \\
& =k \Delta \theta-B \omega
\end{aligned}
$$

The equation with the nonlinearity is:

$$
\tau_{n}= \begin{cases}0, & \Delta \theta<0 \\ k \Delta \theta-B \omega, & \Delta \theta \geq 0\end{cases}
$$

We also note the fact that this equation is also bounded by a region of saturation, which means that the final output torque is:

$$
\tau= \begin{cases}\tau_{n}, & \tau_{n}<265 \mathrm{mNm} \\ 265 \mathrm{mNm}, & \tau_{n} \geq 265 \mathrm{mNm} .\end{cases}
$$

Here $265 \mathrm{mNm}$ corresponds to the maximum stall torque of the motor which is achieved at $100 \%$ duty cycle. This occurs when the calculated value of $\tau_{n} \geq 2^{12}$. 

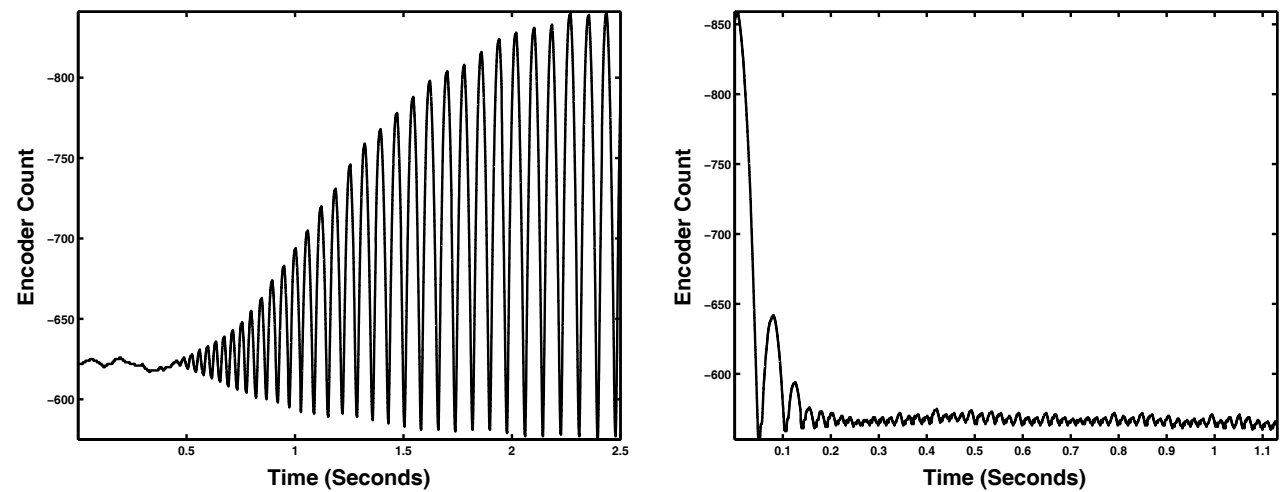

(a) Conventional haptic control system showing (b) Our hybrid haptic control system showing active behavior at the margin of stability, $k=$ active behavior at the margin of stability, $k=$ $2583.28 \mathrm{Nm}^{-1}$ $11624.769 \mathrm{Nm}^{-1}$

Figure 3: Comparison of haptic loops at $\mathrm{B}=0$ (only inherent motor damping is present)

\subsection{Conventional haptic device}

The conventional haptic loop was also implemented for comparison with our haptic loop. To keep the mechanical parameters unchanged for the comparison, the same mechanical setup was used to implement the conventional haptic loop. However the FPGA was programmed to introduce a Zero Order Hold in the forward path. The hold time of the $\mathrm{ZOH}$ was varied in accordance with the sampling frequency desired.

\section{Results}

The results in this section have been arrived at by comparing our modified haptic loop and the conventional digital haptic loop. First we present the results of implementation of the conventional haptic loop. We subsequently compare the performance of the conventional and our modified haptic loop. The comparison is done on the basis of the maximum stiffness that can be achieved in a virtual wall simulation without either system showing active behavior.

Damping present in our haptic device was estimated using the conventional haptic loop. We use the expression [1]:

$$
K \leq \frac{2\left(B+B_{c}\right)}{T}
$$

The device was found to be unstable for the following parameters $K=1372.36 \mathrm{Nm}^{-1}$ and $T=1 \mathrm{~ms}$. From this data we calculate the parameter $B=36.36 \times 10^{-3} \mathrm{Nsm}^{-1}$

While implementing the traditional haptic loop. The device was tested at various sampling frequencies the results of which are shown in Table 1. It can be seen from the table that at higher sampling frequencies a lower value of the computer damping $B_{c}$ is sufficient to stabilize the system.

Our proposed approach using the hybrid haptic loop was found to render passive virtual walls of stiffness of $11624.769 \mathrm{Nm}^{-1}$. This result clearly shows that our hybrid system can render harder walls 
Table 1: Limit of stability at different frequencies

\begin{tabular}{ccc}
\hline \hline Frequency $(\mathrm{Hz})$ & $K\left(\mathrm{Nm}^{-1}\right)$ & $B_{c}\left(\mathrm{Nsm}^{-1}\right)$ \\
\hline 600 & 2583.28 & 2.1527 \\
1000 & 2583.28 & 0.9687 \\
\hline
\end{tabular}

when compared to the conventional haptic loop(Refer Table 1). It is interesting to note that the hybrid system did not show unstable behavior at any $k$ while the experiment as conducted. It settles into limit cycles at high wall stiffness, however it did not show growing oscillations. This may have been due to the fact that we could not set the stiffness to a value high enough to cause unstable behavior.

Figure 3(a) shows the response of the traditional system when instability just appears in the user's interaction with the virtual wall, here $B_{c}=0.9687 \mathrm{Nsm}^{-1}$. It was not possible to record any unstable behavior in the hybrid system, Figure 3(b) shows the response of the device when it starts to exhibit limit cycle behavior. It is to be noted that the maximum stiffness of the wall achieved in case of our modified haptic system is far greater $\left(11624.769 \mathrm{Nm}^{-1}\right)$ than that of the conventional haptic loop implementation $\left(2583.28 \mathrm{Nm}^{-1}\right)$.

Experiments were also conducted to study the effect of computer damping ' $B_{c}$ ' on the stability. It is to be noted that the term $B \omega$ in Equation 7 is obtained by estimating backward differences at a certain sampling frequency. It was seen that the value of computer damping $B_{c}$ did not affect the performance of the hybrid system to a great degree in the passive range of operation. The quantity $B_{c}$ was, however seen to effect the limit cycle behavior of the hybrid system in the non-passive region of operation. An increase in the parameter $B_{c}$ was seen to aggravate the problem of limit cycles. A video of the haptic device in action is available at the following url: http://apm.iitm.ac.in/biomedical/hari/rigidhaptics.wmv

\section{Stability of the asynchronous haptic loop}

We now attempt to find the limit of stability of the analog section of the hybrid haptic loop. The equations controlling the response of a DC motor are:

$$
\tau=K \times i
$$

where $\tau$ is the torque produced by the armature current $i$. The mechanical balance of torques on the rotor yields:

$$
J \frac{d^{2} \theta}{d t^{2}}+B \frac{d \theta}{d t}=\tau
$$

where $\theta$ is the angular position of the rotor. An analysis of the electrical circuit involving the motor inductance ' $L$ 'and armature resistance ' $R$ ' gives us:

$$
L \frac{d i}{d t}+R i=V-K \frac{d \theta}{d t}
$$


In virtual wall implementations the motors in the haptic device are used either in stall or near stall conditions we approximate equation 12 to:

$$
L \frac{d i}{d t}+R i=V
$$

Using equation 10 in equation 13 we obtain:

$$
\frac{L}{K} \frac{d \tau}{d t}+\frac{R}{K} \tau=V
$$

Employing the laplace transform and assuming all initial conditions to be zero:

$$
\frac{\tau(s)}{V(s)}=\frac{K}{L s+R}
$$

Similarly equation 11 transforms into:

$$
\frac{\theta(s)}{\tau(s)}=\frac{1}{J s^{2}+B s}
$$

Equations 15 and 16 allow us to construct the haptic control loop as shown in Figure 4.

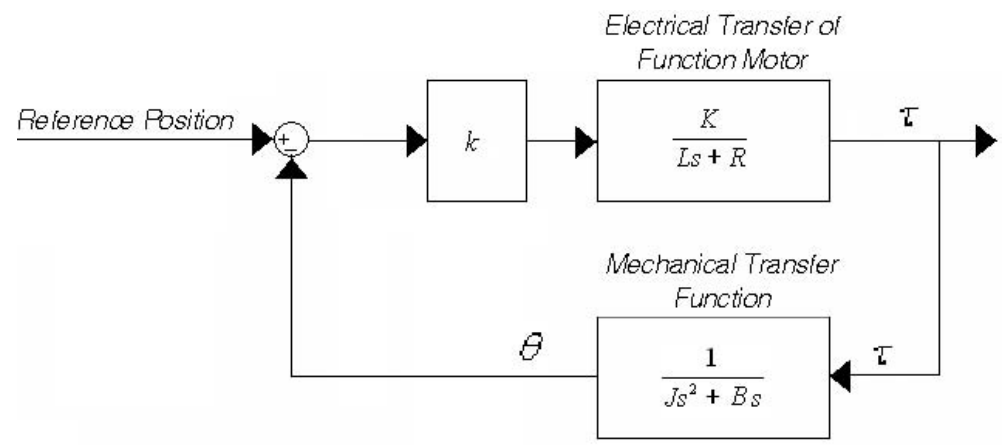

Figure 4: Analog section of the haptic loop

The parameter $k$ controls the stiffness of the virtual wall. We analyze the stability of the haptic loop as the highest value that can be assigned to this parameter. Constructing the forward transfer function $G H(s)$ gives:

$$
G H(s)=\frac{k \times K}{J L s^{3}+(B L+R J) s^{2}+B R s}
$$

It is seen that there exist no poles in the RHS of the s plane for this equation 17. Hence to ensure stability there should be no clockwise encirclements of the point -1 in the s plane. Figure 5 shows an exaggerated plot with clockwise encirclement of the point -1 . The condition for non-encirclement occurs at:

$$
\omega=\sqrt{\frac{R B}{J L}}
$$

using this result the corresponding condition on ' $k$ ' will be:

$$
\frac{k K}{(B R+R J) \frac{B R}{J L}} \leq 1
$$

After some algebra this reduces to:

$$
k \leq \frac{B R}{K}\left[\frac{1}{\tau_{m}}+\frac{1}{\tau_{e}}\right]
$$




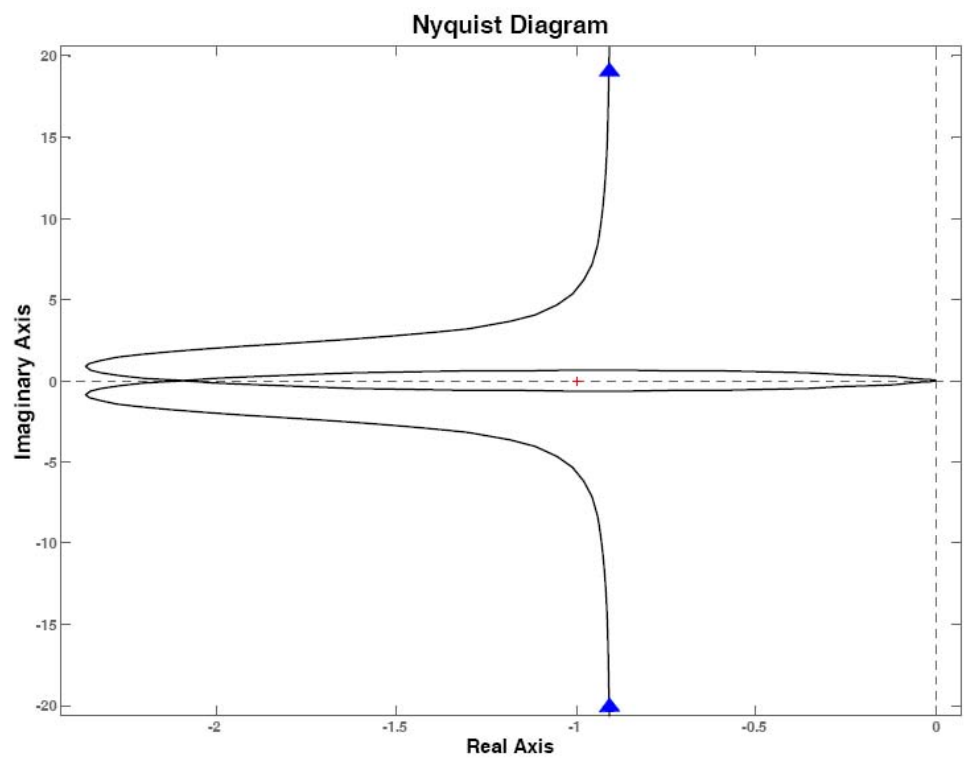

Figure 5: An exaggerated plot of $\mathrm{GH}(\mathrm{s})$ with a clockwise encirclement of the point -1

where $\tau_{m}=\frac{J}{B}$ and $\tau_{e}=\frac{L}{R}$ which are the electrical and mechanical time constants of the system.

Using this equation 20to evaluate the we evaluate the limit of stability of our system. For $B=$ $36.36 \times 10^{-3} \mathrm{Nsm}^{-1}, R=9.61 \Omega, \tau_{e}=87.304 \times 10^{-6} s$ and assuming $\tau_{m} \gg \tau_{e}$, we obtain the limit on $k=75.514 \times 10^{3} \mathrm{Nm}^{-1}$. Because this value of $k$ is very large, it is not surprising that we did not find any unstable behavior of the virtual wall during the course of the experiment. Our hybrid system exhibits limit cycles beyond $k=11624.769 \mathrm{Nm}^{-1}$ on account of the resolution of the encoders.

\section{Discussion}

In the previous section we have shown that our hybrid haptic loop has better performance than conventional haptic loops. Not only did we achieve stiffer walls but also offloaded the force computations to the logic device. It is apparent that freeing the computer of this burden will allow it to use its resources for other compute intensive tasks like graphical rendering, physics modeling etc. We also note that the logic device was instrumental in ensuring fast and noise free performance of the system. Any logic device also has the advantage that it can be interfaced in a very convenient manner to a digital computer. Therefore any changes in stiffness or damping can be easily implemented. Perhaps the only drawback of using the logic device based system is the amplitude quantization that is required for numerical operations. In general, haptic devices use encoders for the purpose of position feedback. These encoders by their very nature cause amplitude quantization of the absolute shaft position. Therefore the performance of any system is more dependent on the amplitude quantization in the encoder than the logic device. 


\section{Future Work}

This hybrid method of implementing the haptic loop opens up many exciting avenues of research. A logical extension of our work is the development of two and three dimensional haptic devices employing similar control systems. We are also exploring implementation of other control paradigms, an interesting problem is the Passivity Controller and Passivity Observer algorithm [7], it is to be investigated whether the use this algorithm can further increase the maximum stiffness of the virtual wall. It is our assumption that research on such continuous time implementations of haptic control systems will eventually result in the development of a Haptics Processing Unit or HPU.

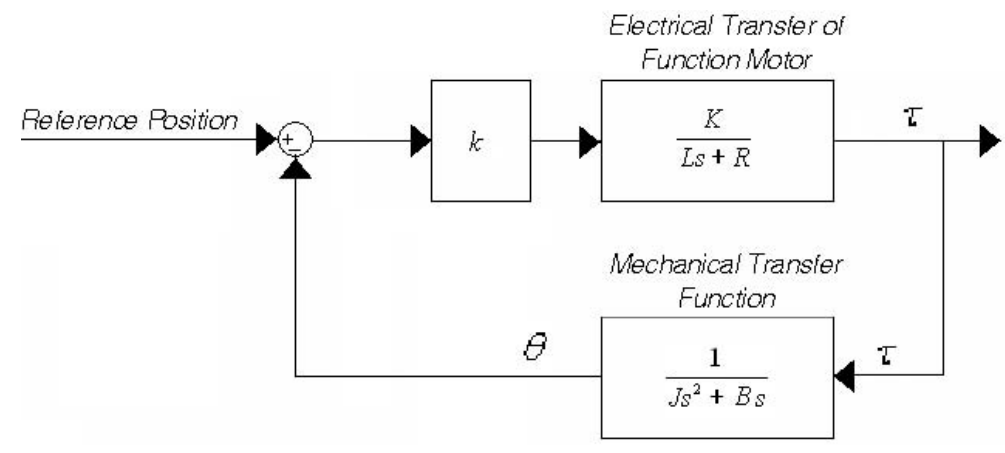

Figure 6: Responses of integrate-and-fire type units.

\section{REFERENCES}

[1] Margaret Minsky, Ouh-Young Ming, Oliver Steele, Frederick P. Brooks Jr., and Max Behensky, "Feeling and seeing: issues in force display", in SI3D '90: Proceedings of the 1990 symposium on Interactive 3D graphics, New York, NY, USA, 1990, pp. 235-241, ACM Press.

[2] Abbott and Okamura, "Effects of position quantization and sampling rate on virtual-wall passivity", IEEE Transactions on Robotics, vol. 21, no. 5, 2005.

[3] Brian E.Miller, J.Edward Colgate, and Randy A.Freeman, "Passive implementation for a class of static nonlinear environments in haptic display", in IEEE International Conference on Robotics and Automation. 1999, IEEE.

[4] Gillespie and Cutkosky, "Stable user-specific haptic rendering of the virtual wall", ASME Dynamic Systems and Control, vol. 58, no. Cutkosky96, pp. 397-406, 1996.

[5] J. Edward Colgate, Paul E. Grafing, Michael C. Stanley, and Gerd Schenkel, "Implementation of stiff virtual walls in force-reflecting interfaces.", in $V R, 1993$, pp. 202-208.

[6] Miller, Colgate, and Freeman, "On the role of dissipation in haptic systems", IEEE Transactions on Robotics, vol. 20, no. 4, 2004. 
[7] B.Hannaford and J.H.Ryu, "Time domain passivity control of haptic interfaces", IEEE Transactions on Robotics and Automation, vol. 18, no. 1, pp. 1-10, February 2002.

[8] Kyungno Lee and Doo Yong Lee, "Multirate control of haptic interface for stability and high fidelity", in $S M C$ (3), 2004, pp. 2542-2547.

[9] Diolaiti, Niemeyer, Barbagli, and Salisbury, "Stability of haptic rendering: Quantization, discretization, time-delay and coulomb effects", IEEE Transactions on Robotics, vol. 22, no. 2, pp. 256-268, 2006.

[10] Masayuki Kawai and Tsuneo Yoshikawa, "Haptic display with an interface device capable of continuous-time impedance display within a sampling period", in IEEE/ASME TRANSACTIONS ON MECHATRONICS, 2004, vol. 9.

[11] G. Niemeyer, N. Diolaiti, and N. Tanner, "Wave haptics: Encoderless virtual stiffnesses", in International Symposium of Robotics Research, 2005. 